\title{
RESEARCHING THE POSSIBILITIES AND ATTITUDES TOWARDS EXPANDING THE PROFESSIONAL COMPETENCES OF RADIOGRAPHERS IN NORTH-EASTERN BULGARIA
}

\author{
Mariana Zhivkova Yordanova ${ }^{1 *}$ \\ *1 Medical University „Prof. Dr. Paraskev Stoyanov “Varna e-mail: kupenova@ gmail.com
}

*Corresponding Author: -

E-mail: kupenova@gmail.com

\begin{abstract}
: -
The radiographer's profession holds a significant place among the healthcare professionals in Bulgaria. Applying the medical imaging methods and techniques, performing the nuclear medicine tests and the radiotherapy procedures would be unthinkable without the participation of highly competent radiographers. The introduction of new high-tech equipment and technologies as well as of diagnosing and treating methods pose new challenges to the radiographers who now have to adopt new knowledge and skills and to perfect their competences in order to respond to the challenges of modern healthcare. This study aims at establishing the attitudes and tendency of practicing radiographers towards expanding their professional competences in their field of practice. Documentary, sociological and statistical methods have been used. Sources related to the topic have been researched. The study presents the opinion of 182 radiographers practicing in the North-Eastern region of Bulgaria regarding the attitudes and the tendency to expand the professional competences of radiographers. The interviewees have been distributed as follows: 137 radiographers (75.27\%) practicing in the field of medical imaging, 42 (23.08\%) - in radiotherapy and 3 (1.65\%) in the field of nuclear medicine. The data has been processed mathematically and statistically and the results have been presented as graphs. The study showed that the radiographers from all three fields of practice and within the age group 22-40 years (professional experience up to 15 years) are more willing to expand their professional competences in the field of practice but after additional training compared to the age group 40-60 years (professional experience more than 15 years) who consider the competence they already possess allows them to perform the assigned tasks and that adopting new competences would increase their work load. The data obtained from the study in terms of the field of practice is quite interesting. The radiographers practicing in the field of radiotherapy are more willing to adopt new competences compared to those practicing in the field of medical imaging and nuclear medicine. This is due to some extent to the fact that radiographers working in the field of medical imaging have to deal with more work load, often in emergency situations and this would make difficult assuming new responsibilities.
\end{abstract}

Key words: professional competences, radiographers, medical imaging, nuclear medicine, radiotherapy, attitudes, possibilities

\section{(우 (\$) (1)}




\section{INTRODUCTION}

The radiographer`s profession holds a significant place among the healthcare professionals in Bulgaria. The fields of practice of radiographers in Bulgaria are medical imaging, nuclear medicine and radiotherapy. They are characterised with using high-tech medical equipment for diagnostics and treatment which requires excellently prepared specialists with acquired knowledge and skills (competences) to apply all new methods and techniques in the relevant fields in order to respond to the challenges of modern healthcare. Adopting the basic competences is set in the educational programmes of medical colleges where these specialists study however practice shows that the competences to be adopted are not equal in all three fields of practice and this imposes the necessity of additional training for the fields of nuclear medicine and radiotherapy. The insufficient preparation is due to the smaller number of tuition hours for subjects "Nuclear Medicine" and "Radiotherapy" envisaged in the Uniform State Requirements for Acquiring the Radiography Profession, a principle that is also applied for the educational plans for the specialty.

\section{Aim}

This research aims at establishing the attitudes and tendency of radiographers practicing in North-Eastern Bulgaria towards expanding their professional competences in their field of practice.

\section{Material and methods}

In order to accomplish the goal we designed specific questions concerning the direct work in the relevant field. We offered to radiographers practicing in the three fields - medical imaging, nuclear medicine and radiotherapy in North-Eastern Bulgaria to participate in the inquiry voluntarily in order to establish their possibilities, attitudes and tendencies to expand their professional competences in the respective field of practice. The total number of radiographers willing to participate was 182 distributed as follows: $137(75.27 \%)$ radiographers practicing in the field of medical imaging $42(23.08 \%)-$ in radiotherapy and $3(1.65 \%)$ in nuclear medicine. The small number of interviewees from the Nuclear Medicine sector is due to the fact that this sector is developed only at the University Hospital in Varna for the whole North-Eastern region. We processed the obtained results mathematically and statistically and we present them as graphs.

\section{Results}

The radiographers participating in the research have been distributed into three groups depending on the field of practice and their professional experience in the relevant sector. Figure: 1 shows their distribution as follows:

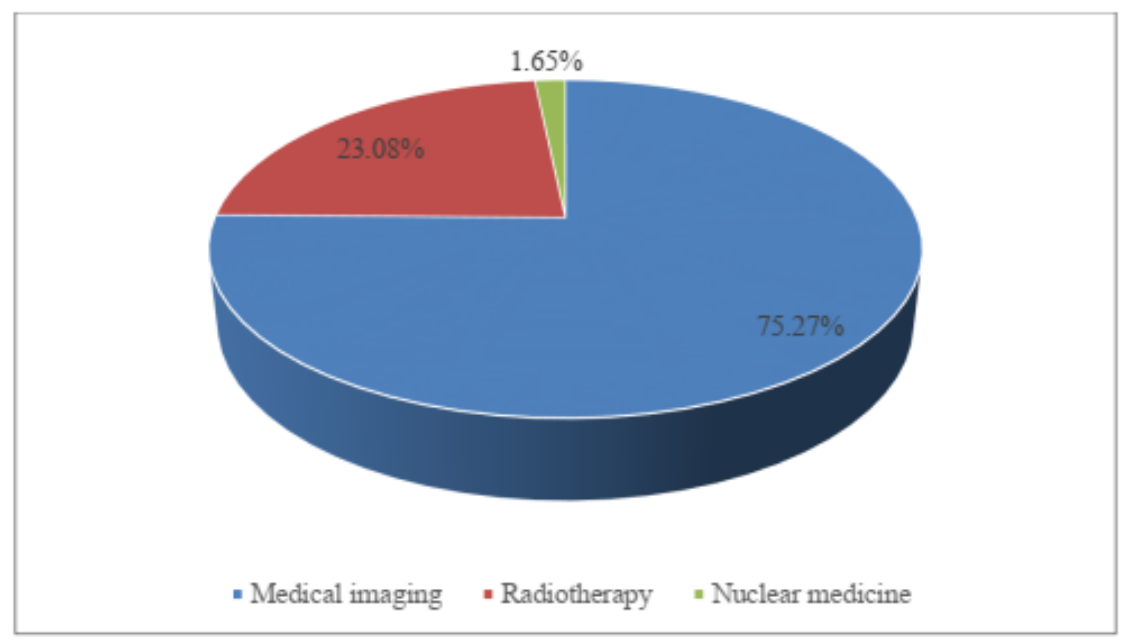

Figure: 1. Distribution by field of practice

Regarding their professional experience the participants in the study have been distributed into two groups: professional experience up to 15 years and a second group - professional experience more than 15 years, and distributed as follows: more than the half of the radiographers practicing in the field of medical imaging (56.93\%) have experience up to 15 years, and $43.07 \%$ - experience of more than 15 years. The ratio of radiographers practicing in the field of radiotherapy with experience up to 15 years to those with experience more than 15 years is $73.81 \%$ to $26.19 \%$, and the ratio in sector Nuclear Medicine is respectively $66.67 \%$ to $33.33 \%$, the majority being those with experience up to 15 years. 


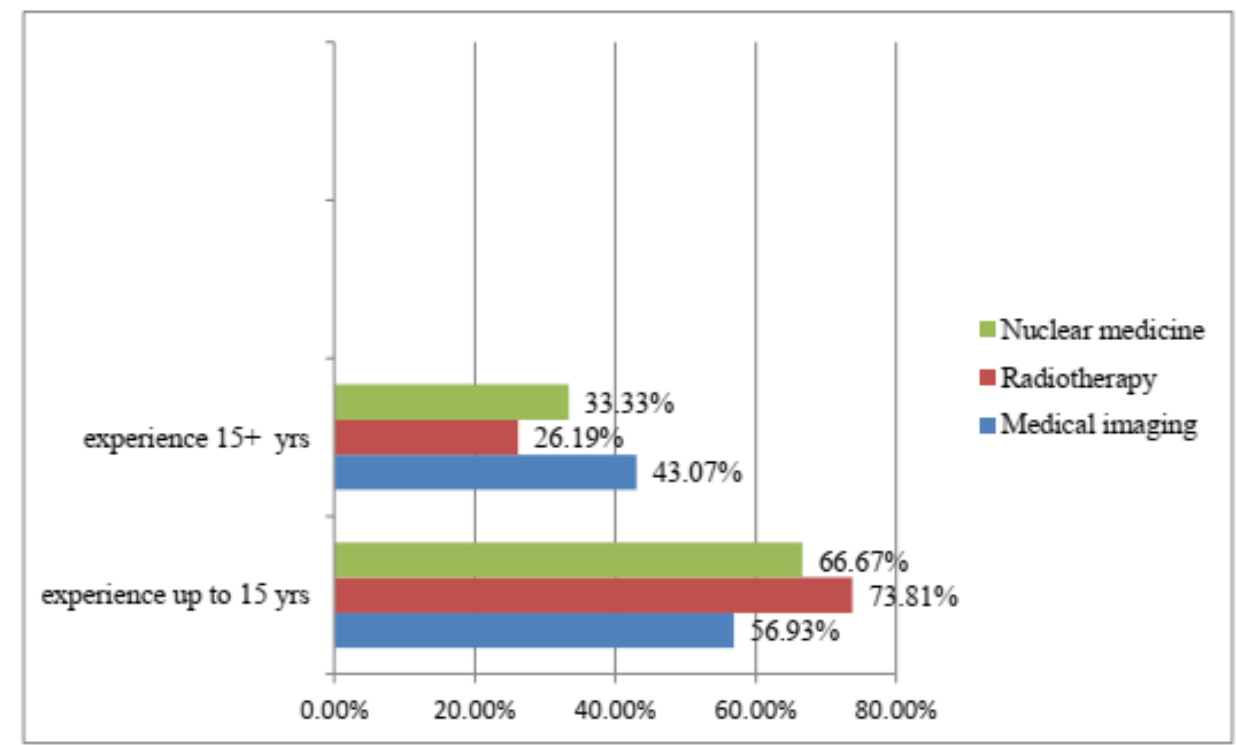

Figure: 2 Distribution by professional experience

Due to the different nature of work of practicing radiographers, questions related to the specifics of their work have been asked. The group practicing medical imaging was asked to answer to the question: "Do you think that radiographers have sufficient competence to conduct ultrasound scan independently?" The respondents were given the possibility to offer their suggestions on this matter. Nearly $16 \%$ of the interviewed radiographers with professional experience up to 15 years expressed certainty in their competence and consider they could handle the examination, while $84 \%$ of the same group and $100 \%$ of the group having $15+$ years of experience think that they don't have the competence or it is insufficient for conducting the ultrasound scan by themselves. The respondents from both groups agree on the suggestion for postgraduate training including a further opportunity for independent conduction of ultrasound scan by the radiographers.

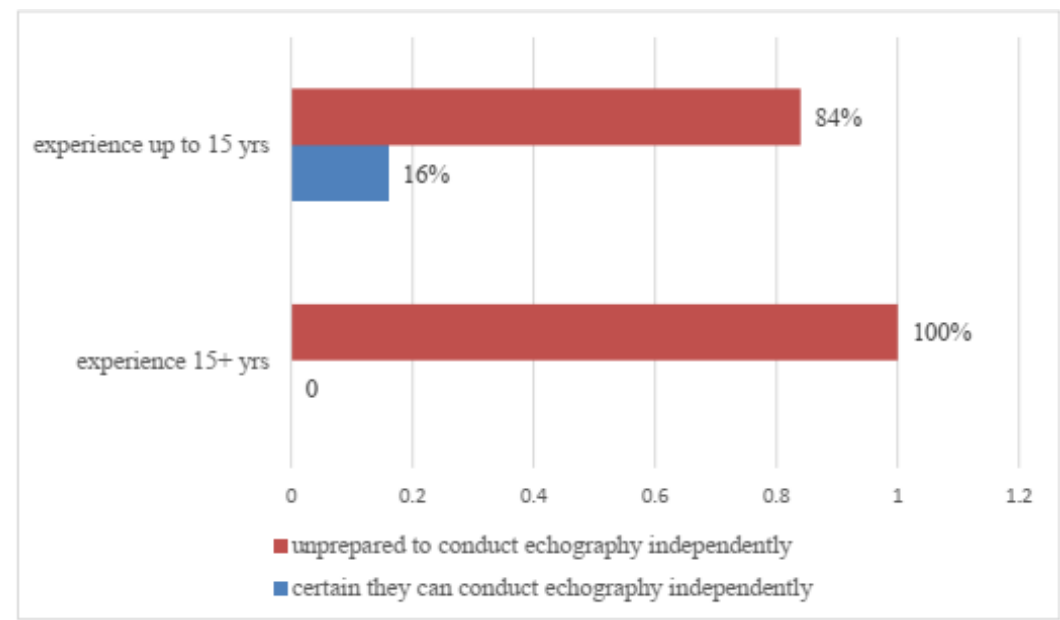

Figure: 3 Competence to conduct independently an ultrasound scan

Respondents were given the possibility to specify the reason they don' $t$ feel prepared enough if they had answered "I am not competent". The group of radiographers with experience $15+$ years indicated as a reason the fact that they studied ultrasound within their education at medical colleges as general methodology only and not like separate subject with the ultrasound anatomy, the practical training controlled by a specialist, the diagnostic options of the ultrasound methodology, the adoption of algorithms for conducting ultrasound scans which are specific for this field. Radiographers with experience up to 15 years have studied ultrasound within 100 tuition hours which are considered insufficient for mastering the methodology in detail, thus making them feel insecure to conduct such examinations independently. For radiographers with $15+$ years of experience the methodology was not intended for studying that is why they lack competences in this field.

We asked the radiographers practicing in the field of nuclear medicine: "Do you think that a radiographer practicing in the field of nuclear medicine could assist the doctor in making the diagnosis if they are given the opportunity to mark in a suitable way the presence of potential pathology within the conducted examination?"

It is noticeable that two of the three respondents practicing in the field of nuclear medicine and having experience up to 15 years would not take the responsibility to commit making a statement on the presence of potential pathology and they consider that this can be applied after further training, while the other respondent with $15+$ years of experience considers that the experience gained by practice could be useful in interpreting some findings. According to that respondent this will give the doctor the opportunity to process larger volume of tests for a shorter period of time. 


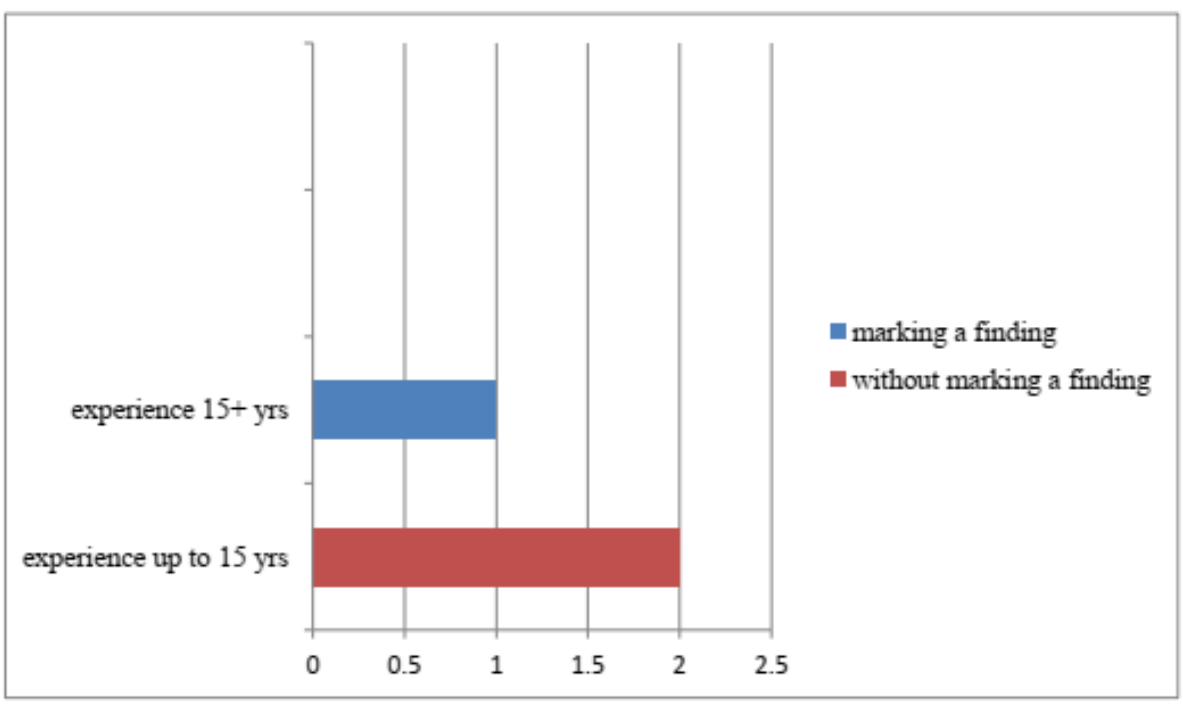

Figure: 4 Expanding the competences by marking findings

All respondents practicing in the field of nuclear medicine consider that adopting additional competences shall give them the opportunity for better performance.

Due to the nature of practice in the field of radiotherapy which is associated to treatment and not with diagnostics of diseases we asked the radiographers working in that sector and we set the aim to establish their possibilities and attitudes towards expanding their professional competences by involving them more actively within the radiotherapy process planning stage which at the moment is performed by the medical physicists. We asked the radiographers practicing in the field of radiotherapy: "Would you accept including into your functional obligations the stage of planning the radiotherapeutic procedures?" Positive answer "Yes, after further training" gave $87.5 \%$ of the respondents of the group (experience up to 15 years), and $12.5 \%$ of the respondents from the same group do not wish to involve in the process even after being trained. Regarding the other group of practitioners with $15+$ years of professional experience $70 \%$ of them consider planning is not in their competences and they do not wish to conduct it even after the provisioning of additional training and barely $30 \%$ of the same group share that this activity is interesting for them and if trained they could conduct it.

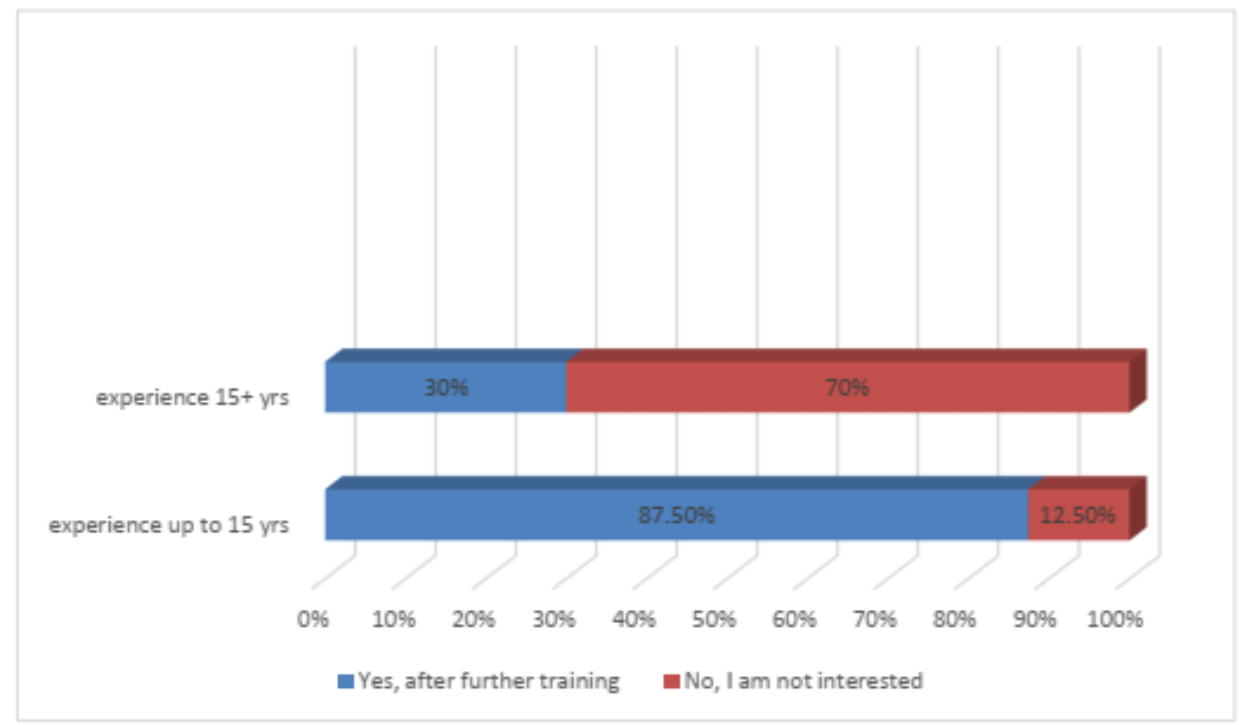

Figure: 5 Participation in planning the radiotherapeutic process

\section{Conclusions}

Unfortunately, from the conducted study and after analysing the obtained results we can conclude the following:

1. In Bulgaria ultrasound is conducted only by physicians specialising in certain fields of medicine and the radiographers with experience up to 15 years, although having studied ultrasoundy, do not feel competent enough to perform it independently as the envisaged number of tuition hours for studying this subject is insufficient for independent practice, and for the practitioners with $15+$ years of experience the subject has not been envisaged for studying within their educational plan at all so for them this is activity requires detailed preparation. The suggestions of all practitioners of medical imaging gather around the introduction of postgraduate training on this subject which shall give the right to practice ultrasound scansy independently. 
2. The radiographers practicing in the field of nuclear medicine have the attitude and tendency to expand their competences through marking findings, this is logically relevant for the radiographers with more extensive professional experience which gives them self-esteem, while those with professional experience up to 15 years still have a lot to learn from practice and share that they may involve themselves in the given opportunity but only after additional training and practice.

3. Significant percentage of the radiographers practicing in the field of radiotherapy are willing to acquire and apply new competences, including involving in new functional obligations but they also stress the need of additional training.

Conclusion: Based on the obtained results from the research we have established that radiographers practicing in the three fields and within the age group 22-40 years (experience up to 15 years) are more willing to expand their professional competences in the field of practice but after additional training compared to the radiographers from the age group 40-60 years (15+ years of experience) who consider the competence they possess allows them to execute the assigned tasks and adopting new competences is considered a way to increase their work load. The data obtained from the study regarding the field of practice are interesting. The radiographers practicing in the field of radiotherapy are more willing to adopt new competences compared to those practicing in the fields of medical imaging and nuclear medicine. In our opinion this is due to the fact that the radiographers practicing in the field of medical imaging have to deal with bigger work load, often in emergency situations and this would hinder the adoption of new responsibilities. We can say for certain that in all three fields of practice exist opportunities to expand the professional competences but at this stage the attitudes of practicing radiographers are limited which gives reason to undertake actions towards increasing the motivation at an early stage, during their college education and towards making corrections in the educational programmes for radiographers.

\section{Bibliography}

[1].Educational documents for the specialty "Radiographer" at the Medical College - Varna

[2].Ordinance No. 1 of 8 February 2011 on the professional activities which nurses, midwives, associated medical specialists and health assistants can perform as designated or independently, SG, issue 15 of 18.02.2011.

[3].Ordinance on the Uniform State Requirements for Acquiring Higher Education in the Specialties in Professional Field" Healthcare" for Educational and Qualification Degree "Professional Bachelor", adopted by decree No. 238 of the Council of Ministers of 26 September 2008, SG, issue 87 of 7.10. 2008.

[4].EFRS Survey 3. Radiographer Societies in Europe. June 2015.

[5].HCPC (health \& care professions council). Standards of proficiency - Radiographers. 28 May 2013.

[6].Price, Richard Charles. DEVELOPING PRACTICE IN RADIOGRAPHY AND DIAGNOSTIC IMAGING. Oct. 2006. 